\title{
La distribución de las fórmulas rutinarias de reproche en la intervención del hablante
}

\author{
The distribution of the routine formulas of \\ reproach within the speaker's intervention
}

\author{
Bojana Tulimirovic \\ Centro de Magisterio La Inmaculada, Universidad de Granada, Granada \\ bojana@cmli.es
}

ACCESO ABIERTO / OPEN ACCESS

Cita: Tulimirovic, Bojana (2021). La distribución de las fórmulas rutinarias de reproche en la intervención del hablante. Textos en Proceso, 7(2), pp. 24-38. https://doi.org/10.17710/tep.2021.7. 2.2tulimirovic

Editoras: Esperanza Alcaide Lara (Universidad de Sevilla) y Ana Pano Alamán (Università di Bologna)

Recibido: $15 / 10 / 2021$

Aceptado: 12/12/2021

Conflicto de intereses: La autora ha declarado que no posee conflicto de intereses.

Copyright: (c) Bojana Tulimirovic. Esta obra está bajo licencia Creative Commons Reconocimiento 4.0

\section{Resumen}

El principal objetivo de este trabajo es analizar la distribución de las fórmulas rutinarias de reproche dentro de la intervención del hablante, esto es, del sujeto que emplea una fórmula rutinaria concreta con la intención de reprochar las acciones previas de su interlocutor. Así pues, se pretende examinar la posición que ocupa la fórmula (inicial, medial, final o independiente) atendiendo a los criterios de la teoría de la segmentación de la conversación propuesta por el grupo Val.Es.Co. Creemos que la dualidad directivo-expresiva que presenta el acto de habla del reproche hace que las fórmulas usadas para vehicular este acto se realicen en entornos sintácticoenunciativos complejos. Por la misma razón, creemos que la posición más frecuente que ocupan estas fórmulas es la medial. Para desarrollar el estudio, se han escogido tres fórmulas rutinarias de reproche y se han analizado 300 ejemplos distintos del corpus esTenTen18 (Spanish Web18). Los resultados preliminares confirman nuestras dos hipótesis de partida: las fórmulas de reproche se desarrollan en intervenciones complejas reactivas ocupando con más frecuencia la posición medial, lo cual implica que existe una clara tendencia hacia el reforzamiento argumentativo de la fórmula.

Palabras clave: fórmulas rutinarias, reproche, distribución, estructura informativa, intervención.

\begin{abstract}
The main goal of this paper is to analyse the distribution of routine formulas of reproach within the intervention of the speaker, that is, the subject that utilises a specific routine formula in order to reproach previous actions of his/her interlocutor. Thus, the goal is to examine the position that a formula occupies (initial, medial, final or independent) by taking into account the conversation theory criteria
\end{abstract}


proposed by the Val.Es.Co. group. We believe that the directive-expressive duality contained in the speech act of reproach is what makes the formula appear in complex utterances and syntactic environments. For the same reason, we believe that the most frequent position that these formulas occupy is the medial. To carry out the study, we have analysed three reproach formulas and 300 different examples from esTenTen18 (Spanish Web18) corpus. The preliminary results confirm our two hypotheses: the routine formulas of reproach are developed in complex reactive interventions occupying the medial position most often, which implies that there is a clear tendency towards the argumentative reinforcement of the formula.

Keywords: routine formulas, reproach, distribution, informative structure, intervention.

\section{Introducción}

El lugar que ocupan las fórmulas rutinarias (FR, de aquí en adelante) en la lingüística siempre se ha considerado poco prominente (cfr. Alvarado Ortega, 2008; Casares, 1950; Corpas Pastor, 1996), pues estas unidades fraseológicas han estado al margen del interés de los estudiosos durante un dilatado periodo de tiempo. No obstante, son muchas las propuestas que han ido surgiendo a lo largo de los últimos años cuyo principal objetivo es ampliar el conocimiento sobre este grupo de unidades fraseológicas ofreciendo nuevas vías de análisis y proporcionando información valiosa sobre su funcionamiento como enunciados fraseológicos (cfr. Alvarado Ortega, 2008; Amigot Castillo, 2014; Núñez Bayo, 2016 o Tulimirovic, 2021a). En este sentido, el trabajo que se presenta a continuación tiene como objetivo seguir aportando información sobre las FR mediante el análisis de la estructura informativa de las intervenciones en las que aparecen las FR para verificar en qué posición -inicial, medial, final o independiente- suelen realizarse y por qué. Para ello, nos centraremos en las FR de reproche, dado que se trata de un acto complejo en el plano enunciativo (Tulimirovic, 2021b), lo cual nos permite observar el comportamiento de las FR en contextos con más carga informativofuncional.

Si nos detenemos en las propiedades del reproche como acto de habla (Tulimirovic, 2021a, 2021b; Vallejo Zapata, 2021), vemos que son dos vertientes principales las que destacan en la realización de este acto. Por un lado, se trata de un acto expresivo dado que el hablante no está contento con las acciones y/o palabras de su interlocutor y no duda en expresar su desagrado hacia el oyente. Por otro lado, también es un acto directivo, debido a que, aparte de mostrar su disconformidad por las acciones del oyente, el hablante procura que este cambie su actitud o que remedie la situación que ha causado el sentimiento de molestia en un primer término (Tulimirovic, 2021, p. 203).

Esta complejidad que denota la naturaleza de dicho acto nos ayuda a definir nuestra primera hipótesis del estudio: la dualidad directivo-expresiva contenida en el propio acto de habla del reproche hace que las FR que se emplean para vehicular dicho acto se realicen en entornos sintáctico-enunciativos muy complejos. Dicho en otras palabras, creemos que las intervenciones del hablante se caracterizan por la misma complejidad enunciativa, por lo que la FR usada en estos contextos 
normalmente va acompañada por otros enunciados que ayudan a llevar a cabo la tarea dual que describimos. Así pues, la estructura informativa de las intervenciones en las que aparecen las FR de reproche se podría definir como tripartita, entendiéndose por ello cualquier intervención compleja que contenga tres o más enunciados donde la FR se emplee en la posición medial, es decir, en una de las posiciones centrales de la intervención, precisamente por el refuerzo argumentativo que creemos que estas FR precisan.

La estructura que presentamos sugiere que, además de aplicar la FR -que se erige como el elemento central de la intervención- el hablante tiene la necesidad de poner en evidencia las acciones del oyente y así dar paso a la propia FR (al reproche en sí) seguida por la información adicional que ayuda a mostrar su estado de ánimo y su actitud/opinión general tras presenciar los actos del oyente. Creemos que en algunos casos este último enunciado (o bloque de enunciados) incluso puede llevar a la realización de otros actos de habla con más carga expresivo-directiva, como puede ser una amenaza, un insulto, etc.

Con todo, nuestra segunda hipótesis se podría formular del siguiente modo: la posición en la que la FR de reproche actúa con más frecuencia dentro de la intervención del hablante es la medial. Además, la propia intervención del hablante se realiza de manera reactiva y no iniciativa, pues tiene que haber un contenido explícito previo (contenido proposicional) que desate el reproche.

\section{Consideraciones teóricas}

Son muchas las líneas de investigación cuyo objetivo es definir la estructura informativa (information packaging) de $\operatorname{los}$ enunciados $^{1} \mathrm{y}$ determinar de qué manera se segmenta la conversación y qué cambios conlleva con respecto al plano comunicativo. Según Robles Sabater y Bertomeu-Pi (2018), algunas de las más productivas en el ámbito de la lengua española tienen que ver con la posición de los constituyentes del discurso (Ariza, 1978; Contreras, 1978), el orden de palabras en español escrito y hablado (Contreras, 1978; Hernando, 2005; Padilla, 2005; Villalba, 2010), el concepto de la dislocación o la focalización, la organización del discurso (Fuentes, 2007, 2014), los marcadores del discurso (Cortés y Camacho, 2005; Portolés, 2010; Salameh et al., 2018) y también el modelo de segmentación de la conversación oral donde priman las propuestas del Grupo Val.Es.Co. o trabajos que parten de la misma base (Briz, 1998a, 1998b, 2000b, 2000a, 2003; Briz y Grupo Val.Es.Co., 2000; Briz e Hidalgo, 1998; Grupo Val.Es.Co., 2002, 2014; Hidalgo y Padilla, 2006; Latorre Garcia, 2018; Padilla, 2006).

En este trabajo, tomaremos como referencia teórica precisamente las aportaciones del grupo Val.Es.Co., pues creemos que el modelo que proponen es una herramienta sólida que nos puede ayudar a conocer el dominio de una FR dentro de la conversación y también su posición, al tiempo que verifica el grado de independencia enunciativa que esta tiene dentro de intervenciones complejas.

\footnotetext{
${ }^{1}$ Tal y como recuerdan Robles Sabater y Bertomeu-Pi (2018: p. 1), este concepto se remonta a las indagaciones de Halliday (1967), entre otros, para quien el análisis de los enunciados debería centrarse no en "las relaciones sintácticas que mantienen sus constituyentes, sino en la posición que estos ocupan dentro del discurso" (Halliday, 1967 apud Robles Sabater y Bertomeu Pi, 2018: p. 1).
} 
Tabla 1. Sistema de unidades (Grupo Val.Es.Co.)

\begin{tabular}{|l|l|l|l|}
\hline \multirow{2}{*}{ NIVEL } & \multicolumn{3}{|c|}{ DIMENSIONES } \\
\cline { 2 - 4 } & \multicolumn{1}{|c|}{ ESTRUCTURAL } & \multicolumn{1}{|c|}{ SOCIAL } & INFORMATIVA \\
\hline Dialógico & $\begin{array}{l}\text { discurso } \\
\text { diálogo } \\
\text { intercambio }\end{array}$ & alternancia de turnos & \\
\hline Monológico & $\begin{array}{l}\text { intervención } \\
\text { acto }\end{array}$ & turno & subacto \\
\hline
\end{tabular}

Fuente: Grupo Val.Es.Co. (2014, p. 14)

Como se puede observar, la propuesta del grupo Val.Es.Co. se basa en tres dimensiones/órdenes (social, estructural e informativa) y ocho unidades (discurso, diálogo, intercambio, alternancia de turnos, intervención, turno, acto y subacto) a las que se añaden cuatro posiciones (inicial, medial, final e independiente) no recogidas en la tabla 1. Además, cuenta con dos niveles -dialógico y monológico-, pues, tal y como afirma el Grupo, el límite entre estos dos niveles es lo que representa la cuestión principal de este modelo.

Del mismo modo, el sistema de unidades obedece a una estructura interna dado que está organizado por niveles y luego agrupado por dimensiones. Se establece una jerarquización ya que "las unidades del orden inferior son los constituyentes inmediatos de la unidad del orden superior" (Grupo Val.Es.Co., 2014: 13). Así pues, se puede decir que una intervención -la unidad monológica máxima según los presupuestos de la Escuela de Ginebra- es más amplia que un turno -la unidad monológica máxima según los presupuestos del Análisis Conversacional $-^{2}$, pues "todo turno es, al mismo tiempo, una intervención, pero no toda intervención puede constituirse en turno" (Grupo Val.Es.Co., 2014, p. 16). La disparidad dimensional de uno y otro se debe principalmente a la contribución que estos hacen a la conversación: mientras que la intervención provoca una reacción normalmente de tipo lingüístico- ante un mensaje, un turno supone la aceptación del mensaje emitido por el resto de los interlocutores contribuyendo de esta manera a la progresión de la conversación. El hecho de aceptar un mensaje ya es una reacción en sí, pero no lo es en sentido inverso; una reacción no necesariamente significa que el interlocutor haya aceptado o reconocido dicho mensaje.

Al margen de esta distinción angular, cabría recordar que la intervención es la unidad monológica máxima en el plano estructural y el turno lo es en el plano social: la intervención "forma parte del armazón -lingüístico o sintáctico- de la conversación" (dimensión estructural) , p. 113) y el turno "pertenece a la relación que se establece entre hablante y oyente(s) y necesita del reconocimiento de los demás para instaurarse" (Grupo Val.Es.Co., 2014).

Por lo que respecta a esta investigación, hemos de reiterar cuál es su principal objetivo: ver con qué frecuencia aparecen las FR de reproche en distintas posiciones

\footnotetext{
${ }^{2}$ A este respecto, son claras las diferencias entre las dos corrientes teóricas en cuanto a lo que representa la unidad monológica máxima, por una parte, y la unidad dialógica mínima, por otra parte. Mientras que para la Escuela de Ginebra la unidad monológica máxima es la intervención y la unidad dialógica mínima el intercambio, para el Análisis Conversacional, la unidad monológica máxima es el turno, mientras que la unidad dialógica mínima es el par adyacente (Grupo Val.Es.Co., 2014, p. $14)$.
} 
dentro de la unidad monológica máxima (intervención/turno), comprobando así la complejidad enunciativa en la que se realizan. Quedan, por lo tanto, al margen del interés de este trabajo las posibles reacciones del interlocutor, sea eso una aceptación del mensaje, ergo, un turno o un mero cambio de emisor, ergo, una intervención. Si bien es cierto que un reproche -o incluso cualquier tipo de FTA (Face-Threatening-Acts, según Brown y Levinson, 1978, 1987)- posiblemente tenga efectos perlocutivos concretos en el oyente, insistimos en que no es relevante para este estudio en concreto.

Además, hay que tener en cuenta que los corpus de fuentes escritas pueden suponer una dificultad añadida para el análisis de este tipo ${ }^{3}$, sobre todo a la hora de demarcar una reacción -lingüística o no lingüística- de una aceptación, tal y como apunta el grupo Val.Es.Co. Por sendas razones, aquí aplicaremos los dos términos de manera indistinta.

Antes de proceder con cuestiones metodológicas planteadas en este estudio, convendría perfilar algunos aspectos que atañen al propio acto de habla de reproche, pues es el objeto del trabajo, al menos de manera indirecta. Tal y como decíamos en el primer apartado, se trata de un acto directivo-expresivo que se inscribe en la red de actos que amenazan a la imagen pública del oyente, por lo que se denomina FTA o, como indicamos anteriormente, Face-Threatening-Act siguiendo los postulados de Brown y Levinson, $(1978,1987)$. La convergencia de estos dos agentes (expresivo y directivo) apunta a una clara complejidad que el reproche acarrea en el plano comunicativo (Tulimirovic, 2021a, 2021b).

Además, el reproche es unidireccional —del hablante hacia el oyente-, directo — el hablante apela al oyente de manera directa y abierta - y directivo existe un claro deseo de que el oyente remedie la situación que ha causado la decepción o el sentimiento de molestia en el hablante en una primera instancia-, volviendo así a la naturaleza dual que se le asigna (Tulimirovic, 2021b).

Este binomio, junto con las directrices teóricas de (Searle, 1969, 1976, 1979), es el que utiliza Tulimirovic (2021a, 2021b) para definir las condiciones de uso del acto en cuestión. Así pues, la condición de contenido proposicional se entiende como un "hecho pasado $A$ llevado a cabo por $O$ posiblemente como resultado de una reiteración del $A$ o una acumulación de hechos parecidos" (Tulimirovic, 2021a, p. 203). Una vez presenciado este hecho pasado, el hablante actúa con dos objetivos en mente: mostrar a) su desagrado/disconformidad por lo que acaba de experimentar y b) que el oyente es el culpable por lo ocurrido y que debe, por tanto, remediar la situación, esto es, debe conseguir que el sentimiento de molestia en el hablante amaine (Tulimirovic, 2021a, p. 203).

En línea con las condiciones preparatorias, se puede decir que son dos las condiciones esenciales que definen el acto: a) "cuenta como una expresión de disconformidad/desacuerdo ante $A$ hecha por $O$ ", y b) "cuenta como un intento de hacer que $O$ haga $A$ (o que $O$ cambie $A$ )". No obstante, Tulimirovic (2021a, 2021b)

\footnotetext{
${ }^{3}$ Según Vela Delfa y Jiménez Gómez (2011), el carácter de los intercambios de turnos en mensajes escritos y mediatizados por ordenador "les confiere una naturaleza específica que requiere descripciones particulares, puesto que la realidad nos demuestra que estas conversaciones funcionan, aunque con mecanismos de alternancia y organización de turnos propios y diferentes a los de otros entornos conversacionales" (Vela Delfa y Jiménez Gómez, 2011, p. 137).
} 
sostiene que existe una leve prevalencia del componente directivo en el acto, lo cual ayuda a definir la condición de sinceridad como $H$ desea que $O$ haga/remedie $A$.

\section{Metodología}

Para poder llevar a cabo el análisis dentro de los límites de este artículo, se han examinado tres FR que cumplen la función de reproche. Para ello, nos hemos servido del trabajo de Tulimirovic (2021a) quien analiza un grupo de 15 FR que se emplean para vehicular algunos de los FTA, entre los cuales destaca precisamente el reproche. Nos referimos a las siguientes FR: qué te crees, que no te enteras y a quién se le ocurre.

Para recuperar los ejemplos y así explorar la aparición de las FR en contextos reales, hemos extraído secuencias del corpus escrito esTenTen18, Spanish Web 2018 (dentro del grupo de los corpus textuales TenTen 18), al cual hemos podido acceder a través de la herramienta de análisis Sketch Engine ${ }^{\circledR}$. Son dos razones principales por las que hemos escogido este corpus en concreto: a) cuenta con 19,6 billones de palabras, tanto de español peninsular como de español americano, más que ningún otro corpus de estas características, y b) está formado por más de 57 millones de textos (comentarios en foros, prensa en línea, blogs, artículos de opinión, etc.) recopilados de la red por lo que incluye fuentes distintas entre sí, lo cual hace que tengamos un banco de ejemplos muy heterogéneo, tanto a nivel variacional como a nivel funcional. Asimismo, la herramienta Sketch Engine ${ }^{\circledR}$ proporciona un contexto muy amplio para cada una de las consultas. En este caso, hemos optado por la búsqueda KWIC (Key Word in Context), esto es, la concordancia KWIC, precisamente por la facilidad que ofrece de observar el contexto y el cotexto en el que se emplea la FR analizada.

Tal y como se desprende de estas líneas, hemos optado por un corpus de fuentes escritas, a pesar de que el sistema de unidades sobre el cual se basa nuestro marco teórico versa sobre el lenguaje oral. Aun así, creemos que muchas de las unidades que se recogen en la propuesta teórica del Grupo Val.Es.Co. sirven también para examinar las fuentes escritas, siempre y cuando su identificación no se vea impedida por los errores o incongruencia propios de la ortografía y de la sintaxis. Además, hemos de tener en cuenta que, desde una perspectiva concepcional (Koch y Oesterreicher, 1985, 1990, 2001, entre otros), la dimensión variacional "oral/escrito" se percibe como un continuo gradual entre la inmediatez y la distancia comunicativa, de modo que gran parte de los tipos textuales incluidos en esta base de datos de la que hemos extraído los ejemplos para el análisis estarían más cerca del polo "oral".

En total, hemos extraído y analizado 300 ocurrencias diferentes, en función de la frecuencia con la que aparecen las FR en cuestión dentro del corpus consultado. Como las tres FR examinadas se registran con un índice de frecuencia mayor a $100^{4}$, se han analizado solo los primeros 100 casos porque creemos que es un número representativo. Sin embargo, tras un primer estudio, nos hemos dado

\footnotetext{
${ }^{4}$ La FR qué te crees se registra en 1067 casos (0,05 tokens por millón); la FR que no te enteras se registra en 1548 casos ( 0,08 tokens por millón); la FR a quién se le ocurre se registra en 2643 casos distintos (0,13 tokens por millón). Recuperado de: https://www.sketchengine.eu/estenten-spanishcorpus [30/12/2021].
} 
cuenta de que no todos los usos de las expresiones analizadas se pueden suscribir al dominio fraseológico por no realizarse como unidades pluriverbales institucionalizadas, sino más bien como combinaciones libres de palabras, de manera que hemos optado por excluirlas del análisis posterior. Por esta razón, el número definitivo de ocurrencias estudiadas ha sido 226 (59 para la FR qué te crees, 71 para la FR que no te enteras y 96 para la FR a quién se le ocurre), un número que ha permitido llevar a cabo el estudio satisfactoriamente, pese a que ha sido inferior al número previamente ideado.

Cada uno de los ejemplos analizados se ha registrado mediante una ficha fraseológica que contiene los siguientes datos: 1) número de token de la FR dentro del corpus, 2) posición que ocupa la FR en la intervención, 3) valor funcional del enunciado previo (si procede), y 4) valor funcional del enunciado posterior (si procede). Para facilitar la localización de la posición de la FR y así completar el segundo ítem de la ficha, hemos hecho una distinción entre tres tipos de intervenciones: 1) intervención simple -la FR se realiza de manera independiente, ya que es el único enunciado de la intervención-, 2) intervención bimembre -la FR se realiza o bien en la posición medial o final (pero no medial o independiente), ya que son solamente dos enunciados que componen la intervención, y 3 ) intervención compleja -la FR puede ocupar la posición inicial, media o final (pero no independiente), pues son tres o más enunciados que la componen (tal y como se ha explicado anteriormente)-.

Como se puede percibir, nos hemos centrado en el análisis de las cuatro posiciones y en los componentes estructurales de nivel monológico dentro del marco teórico presentado en el apartado anterior. Dicho esto, no hemos tratado las demás unidades del nivel dialógico ni tampoco la tipología de actos/subactos que desempeñan las FR, puesto que creemos que, al contrario de lo que sostiene Alvarado Ortega (2006), una FR siempre se realiza o bien como un acto independiente -cuando es el único elemento de la intervención - o bien como un subacto sustantivo director - cuando se realiza en una intervención compleja, como es el caso de las FR de reproche-, pues cuenta con su propia fuerza ilocucionaria que la aísla del resto de la intervención.

Por último, cabe añadir que, pese a las dificultades que a veces implica establecer los límites entre un enunciado u otro, especialmente en la comunicación oral (cfr. López Serena, 2012), esto no ha tenido lugar en el caso de las FR por dos razones fundamentales: 1) los límites intrínsecos que presentan las propias FR, en tanto que unidades institucionalizada y con un cierto grado de fijación, y 2) la independencia enunciativa de la que gozan las FR por el hecho de contar con su propia fuerza ilocucionaria y por vehicular actos de habla concretos, o lo que es lo mismo, por ser enunciados fraseológicos. 


\section{Resultados}

\subsection{FR qué te crees}

A pesar de que los diccionarios fraseológicos ${ }^{5}$ lematizan esta FR como una expresión que sirve para "protestar de la actitud errónea" o para "expresar incredulidad, rechazo o negación", Tulimirovic (2021a) considera que se trata de una FR clara de reproche, pues cumple todas las condiciones de este acto descritas en el apartado 2.

Ahora bien, a la hora de examinar su distribución, hemos encontrado lo siguiente:

Tabla 2. Distribución de la FR qué te crees

\begin{tabular}{|c|c|c|}
\hline \multirow{2}{*}{$\begin{array}{c}\text { POSICIÓN DE LA FR EN } \\
\text { LA INTERVENCIÓN }\end{array}$} & \multicolumn{2}{|c|}{$\begin{array}{c}\text { CORPUS SPANISH WEB 2018 } \\
\text { (ESTENTEN18) }\end{array}$} \\
\cline { 2 - 3 } & $\mathrm{n}$ & $\%$ \\
\hline inicial & 24 & 40,7 \\
\hline medial & 31 & 52,5 \\
\hline final & 2 & 3,4 \\
\hline independiente & 2 & 3,4 \\
\hline TOTAL & $59^{6}$ & 100 \\
\hline
\end{tabular}

Los datos demuestran que las posiciones más frecuentes son precisamente la medial y la inicial con un $52,5 \%$ y un $40,7 \%$ respectivamente. Dicho esto, son tan solo $6,8 \%$ de los casos donde la FR se emplea como el único enunciado de la intervención, esto es, en la posición independiente, o en la posición final dentro de los enunciados bimembres o complejos.

Este dato nos da indicios sobre la complejidad enunciativa de la que hablábamos en los aparatados anteriores, lo cual se puede entender mejor si nos fijamos en los siguientes ejemplos $(1,2)$ :

1) ${ }^{7}[\ldots]$ Atsushi me conto lo que te paso y supuse que estarías aquí. - Hablo serio el azabache mientras miraba al borracho miembro de Rakuzan. $</ \mathrm{s}\rangle\langle/ \mathrm{p}\rangle\langle\mathrm{p}\rangle\langle\mathrm{s}\rangle^{8}$ ¿Qué te crees! $</ \mathrm{s}><\mathrm{s}>$ ¡Ni eres mi madre para decirme algo así! -Le corrió la mano -Déjame beber en paz, no vale vivir si Sei-chan no está conmigo... -Hablo deprimido. $</ \mathrm{s}></ \mathrm{p}><\mathrm{p}><\mathrm{s}>-[\ldots]$ ( ${ }^{0}$ token: 17979709764).

2) $[\ldots]</ \mathrm{s}><\mathrm{s}>$ Aquel hombre volvió a clavarme su mirada vacía de muñeco de cristal. $</ \mathrm{s}\rangle</ \mathrm{p}><\mathrm{p}><\mathrm{s}>-$ Tú no vas a llamar a nadie, cerda. $</ \mathrm{s}><\mathrm{s}>$ ¿Qué te crees, imbécil? $</ \mathrm{s}><\mathrm{s}>$ De las podridas como tú se ríe la policía -gritó de pronto abalanzándose sobre mí. $</ \mathrm{s}></ \mathrm{p}><\mathrm{p}><\mathrm{s}>[\ldots]$ (no token: 3758242862).

\footnotetext{
${ }^{5}$ Esta FR se recoge en el Diccionario Fraseológico Documentado del Español Actual (Seco, Andrés, y Ramos, 2009) y también en el Diccionario Fraseológico del Español Moderno (Varela y Kubarth, 2004).

${ }^{6}$ Recordemos que son muchos los casos descartados por no ser ejemplos de construcciones fraseológicos, sino por formar parte de la sintaxis libre. En este caso, de 100 ejemplos analizados, en 59 de ellos la secuencia qué te crees se realiza como una unidad fraseológica.

${ }^{7}$ Todos los ejemplos se han transcrito sin corrección alguna.

${ }^{8}</ \mathrm{s}></$ p $><$ p $><$ s $>$ indica el cambio de interlocutor y el comienzo de una nueva intervención/turno.
} 
En ambos ejemplos, estamos ante una intervención compleja, pues contiene al menos tres enunciados distintos. Sin embargo, la disposición de la FR en el primer ejemplo es distinta a la del segundo porque, mientras ambas sirven para introducir un reproche, en el segundo hay una mención explícita del contenido proposicional ("llamar la policía"), lo cual se puede interpretar como una justificación clara del reproche. En el primer ejemplo, en cambio, el contenido proposicional aparece más tarde en la secuencia ("decirme algo así", donde "así" es la verdadera proposición que ha causado la molestia en el hablante y, consecuentemente, el reproche). Además, el primer ejemplo cuenta también con otro argumento que sirve como una extensión del reproche ("ni eres mi madre para decirme algo aś'”) y también una orden (acompañada por una justificación en forma de una frase condicional). A su vez, el segundo ejemplo contiene una prohibición clara y un juicio de valor acompañado por calificativos negativos ("cerda", "imbécil", "podrida") que consigue elevar el tono y agravar la relación social entre los interlocutores. Sea como fuere, sendas situaciones comunicativas implican un estado de malestar del hablante hacia el oyente donde la FR es el elemento principal de la intervención y donde los demás enunciados sirven para justificarlo.

Algo parecido ocurre en el ejemplo 3 ya que la FR vuelve a aparecer en una intervención compleja precedida y seguida por una serie de enunciados que acompañan al reproche y ayudan a entender el porqué de las acciones del hablante.

3) $[\ldots]</ \mathrm{s}></$ p $><$ p $><\mathrm{s}>$ No, mira los mensajes que yo he escrito. $</ \mathrm{s}></$ p $><$ p $><\mathrm{s}>$ De todos modos sólo me da miedo que te creas esas tonterías. $</ \mathrm{s}><\mathrm{s}>$ No sé qué tienes en la cabeza, pero las infraestructuras son estatales, ¿qué te crees, que hay una "independencia" y vais y cogéis y decidís que "los trenes por "nuestras vías" no pasan" y no pasan, y todos tan tranquilos? $</ \mathrm{s}></ \mathrm{p}><\mathrm{p}><\mathrm{s}>[\ldots]\left(\mathrm{n}^{\mathrm{o}}\right.$ token: 245877455).

Cabe destacar que la secuencia $F R+$ frase subordinada ("que hay una [...]") es el patrón más común en la estructura informativa de esta FR, pues hemos comprobado que se documenta en un 73,2\% de las ocurrencias analizadas. Es más, creemos que la propia frase subordinada introducida por que se podría considerar como el dispositivo indicador de la fuerza ilocucionaria, ya que ayuda a conocer la verdadera proposición del reproche.

\subsection{FR que no te enteras}

La segunda FR de reproche analizada en este trabajo es que no te enteras, aunque, según Tulimirovic (2021a), también puede desempeñar otras funciones comunicativas como desacuerdo. Aparte de compartir el valor enunciativo nuclear, las FR qué te crees y que no te enteras también cuentan con una disposición similar (Tabla 3). 
Tabla 3. Distribución de la FR que no te enteras

\begin{tabular}{|c|c|c|}
\hline \multirow{2}{*}{$\begin{array}{c}\text { PosicIÓN DE LA FR EN } \\
\text { LA INTERVENCIÓN }\end{array}$} & \multicolumn{2}{|c|}{$\begin{array}{c}\text { CORPUS SPANISH WEB 2018 } \\
\text { (ESTENTEN18) }\end{array}$} \\
\cline { 2 - 3 } & $\mathrm{n}$ & $\%$ \\
\hline inicial & 22 & 31 \\
\hline medial & 34 & 47,9 \\
\hline final & 15 & 21,1 \\
\hline independiente & 0 & 100 \\
\hline TOTAL & $71^{9}$ & \\
\hline
\end{tabular}

De nuevo, estamos ante una FR que mayoritariamente actúa en posición inicial (31\%) y medial (47,9\%) con una distribución de enunciados parecida a la FR anteriormente analizada. Es decir, parece ser que existe una cierta predisposición a usarla en conjunción con otros enunciados, situándola así en posición inicial, medial o final del turno ${ }^{10}$. A diferencia de la FR anterior, esta FR no se suele emplear en las intervenciones simples, al menos no en los ejemplos analizados.

4) [...] No habrá ningún futuro para el pueblo trabajador de este país si no derribamos este pilar del poder. . (luego habrá que ir a por los otros. ..) $</ \mathrm{s}\rangle\langle/ \mathrm{p}\rangle\langle\mathrm{p}\rangle\langle\mathrm{s}\rangle{ }_{i}$ Que no te enteras, Elpidio! $</ \mathrm{s}><\mathrm{s}>$ Que estás en España y en este país los ladrones y desfalcadores no van a la cárcel. $</ \mathrm{s}><\mathrm{s}>$ Aquí se empapela a jueces como Garzón o como tú, que queréis juzgar a los pobres corruptos que ostentan el poder de este país. $</ \mathrm{s}><\mathrm{s}>[\ldots]$ ( $\mathrm{n}^{\mathrm{o}}$ token: 215189833).

En el ejemplo 4, el hablante empieza su intervención con una FR a través de la cual introduce su reproche - recordemos que se trata de un intento de apelar al oyente para que remedie sus acciones y así ayudar a que amaine el sentimiento de malestar en el hablante- seguido por una serie de opiniones (con alto grado de ironía) que sirven para justificar la equivocación cometida por el oyente en sus acciones previas.

5) [...] Sigues insistiendo en que hay fotografías en las que hay Skodas Fabias que parecen azules. $</ \mathrm{s}></ \mathrm{p}><\mathrm{p}><\mathrm{s}>$ Joder, chato, es que no te enteras. $</ \mathrm{s}><\mathrm{s}>$ Te he dicho cinco_mil veces que no hay ningún Skoda Fabia que sea gris azulado o celeste. $</ \mathrm{s}><\mathrm{s}>\mathrm{Y}$ vas tu y hala, a meterla otra vez hasta dentro. $</ \mathrm{s}></ \mathrm{p}><\mathrm{p}><\mathrm{s}>[\ldots]\left(\mathrm{n}^{\mathrm{o}}\right.$ token: 1877228367$)$.

Por su parte, en el ejemplo 5 la FR va precedida por la interjección joder que en este caso no sirve para evidenciar la proposición del reproche, sino para manifestar cómo se siente el hablante ante las acciones de su interlocutor, es decir, enfadado e irritado. De este modo, conocemos el componente afectivo de la secuencia, lo cual también ayuda a identificar el reproche introducido a través de la propia FR. No obstante, el resto de los enunciados señalan la proposición del reproche de manera más clara, pues el hablante le ha tenido que "decir cinco mil

\footnotetext{
${ }^{9}$ Véase nota 7.

${ }^{10}$ Esto se puede deber a la fácil adaptación de esta FR a múltiples entornos sintácticos (aparte de otras estructuras, hemos detectado varios ejemplos de la FR en combinación con los verbos ser, parecer o ver en la posición precedente, por lo que la FR incluso se puede realizar como una proposición subordinada sustantiva dentro de una oración compuesta) y también al hecho de que esta FR en concreto presenta un nivel muy bajo de idiomaticidad.
} 
veces" al oyente que "no existen Skoda Fabia que sea gris azulado o celeste". Es decir, es la repetición de las acciones del oyente (pensar que existen coches de estos colores) lo que ha causado el reproche en primer lugar, de manera que toda la intervención sirve para razonar el reproche.

6) $[\ldots]$ mucho futuro en España. $</ \mathrm{s}><\mathrm{s}>\mathrm{El}$ intervencionismo de Bruselas no es respaldado por la mayoría de los europeos, que lo que quieren es más libertad y menos burocracia, y menos socialdemocracia, y menos jacobinismo y menos grados 33 por el Parlamento Europeo. $</ \mathrm{s}><\mathrm{s}>$ iiCarnicero, que no te enteras!! $</ \mathrm{s}></ \mathrm{p}><\mathrm{p}><\mathrm{s}>[\ldots]\left(\mathrm{n}^{\circ}\right.$ token: 161866864$)$

Por último, el ejemplo 6 ofrece una secuencia donde la FR es el último enunciado de la intervención. Igual que en los ejemplos anteriores, hay una serie de argumentos - en este caso, con menor carga emocional - que ofrece el hablante antes de introducir el reproche con el que culmina su intervención.

\subsection{FR a quién se le ocurre}

La última FR escogida para este trabajo es a quién se le ocurre, una FR de reproche que, como hemos podido demostrar, ofrece un panorama distinto en comparación con las FR anteriormente tratadas, sobre todo por la prevalencia de la posición medial sobre el resto de las posiciones examinadas (Tabla 4):

Tabla 4. Distribución de la FR a quién se le ocurre

\begin{tabular}{|c|c|c|}
\hline \multirow{2}{*}{$\begin{array}{c}\text { POSICIÓN DE LA FR EN } \\
\text { LA INTERVENCIÓN }\end{array}$} & \multicolumn{2}{|c|}{$\begin{array}{c}\text { CORPUS SPANISH WEB 2018 } \\
\text { (ESTENTEN18) }\end{array}$} \\
\cline { 2 - 3 } & $\mathrm{n}$ & $\%$ \\
\hline inicial & 20 & 20,8 \\
\hline medial & 66 & 68,8 \\
\hline final & 10 & 0 \\
\hline independiente & 0 & 100 \\
\hline TOTAL & $96^{11}$ & \\
\hline
\end{tabular}

De los 96 ejemplos contabilizados para el análisis, casi un $70 \%$ de ellos incluye la FR en la posición medial de la intervención dejando así la posición inicial en segundo lugar con un escueto $20,8 \%$. Dicho esto, la posición final es, junto con la posición independiente - no localizada en ninguno de los ejemplos analizadosla menos frecuente en intervenciones bimembres y complejas, pues tan solo hemos podido localizar 10 ejemplos en total. Además, parece ser que en esta FR se repite el mismo patrón enunciativo donde prima el reproche acompañado por otros enunciados que ayudan a argumentarlo:

7) [...] Y el traficante de reliquias, también adorador de Hermes, pensó: "Tonto, tonto. $</ \mathrm{s}><\mathrm{S}>$ ¿Si es que a quién se le ocurre? $</ \mathrm{s}><\mathrm{s}>$ Si sabes, tonto el culo, que puede pasar la pasma en cualquier momento, ¿por qué cojones lo haces, subnormal? $</ \mathrm{s}><\mathrm{s}>$ Si no te pillaban, ya les llamaba yo; pero por gilipollas, chaval, por gilipollas extremo [...] ( $\mathrm{n}^{\mathrm{o}}$ token: 448330557$)$.

${ }^{11}$ Véase nota 7. 
En el ejemplo 7, el hablante primero valora las acciones del oyente mediante el calificativo "tonto" que posteriormente reitera añadiendo nuevas formas ("tonto el culo", "subnormal"). Una vez empleada la FR, añade un nuevo enunciado en forma de una frase interrogativa, pero no con la intención de obtener la respuesta por parte del interlocutor, sino para resaltar la incongruencia que suponen las acciones del oyente (la proposición verdadera del reproche). Lo mismo ocurre en el siguiente ejemplo, aunque con menos carga emocional y con la (ligera) intención de salvaguardar la imagen positiva del oyente a pesar del reproche:

8) $[\ldots]</ \mathrm{s}></ \mathrm{p}><\mathrm{p}><\mathrm{s}>$ Dicho esto, usted puede saber perfectamente que no están al mismo nivel. $</ \mathrm{s}><\mathrm{s}>$ ¿A quién se le ocurre pensar que el castellano puede estar en peligro o en situación de casi casi cercenamiento o discriminación en el Estado español? $</ \mathrm{s}><\mathrm{s}>$ ¿Acaso usted tiene problemas por hablar en castellano en Euskadi? $</ \mathrm{s}><\mathrm{S}>$ ¿Acaso tiene algún problema? $</ \mathrm{s}><\mathrm{s}>[\ldots]$ (n ${ }^{\circ}$ token: 316121661$)$.

En primer lugar, el hablante proporciona un argumento sobre el tema principal de la conversación apelando además al razonamiento del oyente ("usted puede saber perfectamente [...]") e introduciendo de este modo el contenido proposicional del reproche que se presenta en el siguiente enunciado ("pensar que el castellano puede estar en peligro"). Le sigue una serie de frases interrogativas que, de nuevo, no son preguntas per se, sino una manera de mostrar el enfado que siente el hablante hacia el oyente por las incoherencias que este manifiesta en sus acciones anteriores.

9) $[\ldots]</ \mathrm{s}><\mathrm{s}>\mathrm{Y}$ esto es exactamente lo que los Padres de la Iglesia y TODOS los católicos, hasta bien entrado en siglo XX creían. $</ \mathrm{s}\rangle</ \mathrm{p}\rangle\langle\mathrm{p}\rangle\langle<\mathrm{s}>$ Pero debe ser que eran muy ignorantes y muy tontos. $</ \mathrm{s}><\mathrm{s}>$ Creer lo que dice la Biblia... ¿a quién se le ocurre?. $</ \mathrm{s}></ \mathrm{p}><\mathrm{p}><\mathrm{s}>[\ldots]$ ( $\mathrm{n}^{\circ}$ token: 13928014965).

10) $[\ldots]</ \mathrm{s}></ \mathrm{p}><\mathrm{p}><\mathrm{s}>-$ i ¿A quién se le ocurre?! $</ \mathrm{s}><_{\mathrm{s}}>$ ¡Puñetero idiota! $</ \mathrm{s}><\mathrm{s}>$ ¡Por los clavos de Cristo! $</ \mathrm{s}><\mathrm{s}>$ ¿Cómo se puede ser tan gilipollas? $</ \mathrm{s}><\mathrm{s}>$ Que Dios me perdone, pero este muchacho me saca de quicio -el sacerdote se persigna y solicita el perdón del altísimo. $</ \mathrm{s}></ \mathrm{p}><\mathrm{p}><\mathrm{s}>[\ldots]$ ( $\mathrm{n}^{\mathrm{o}}$ token: 1509990166).

Son pocos los cambios que se perciben a nivel enunciativo dentro de la estructura informativa de la FR cuando esta ocupa la posición final (ejemplo 9) y la posición inicial (ejemplo 10) de la intervención. En ambos casos, hay una crítica hacia las actitudes o acciones del oyente que, con más o menos ímpetu, lo exterioriza dando razones para ello.

Los resultados que atañen a esta FR y también a las dos FR previamente analizadas indican que estamos ante patrones enunciativos bastante parecidos, pese a que en algunos casos -la FR a quién se le ocurre, por ejemplo- las frecuencias de la aparición de la FR en distintas posiciones pueden variar con respecto a otras dos unidades fraseológicas. Aun así, reiteramos que las tres FR se comportan de manera casi idéntica, lo cual se atribuye claramente a su valor funcional.

\section{Conclusiones}

El presente estudio sobre las FR de reproche y su distribución dentro de las intervenciones del hablante ha arrojado alguna luz sobre ciertos aspectos poco estudiados en la lengua española con respecto a este grupo de unidades 
fraseológicas. Pese al carácter sucinto del trabajo, creemos que ha permitido conocer el comportamiento de estas unidades en entornos complejos y así aproximarnos a su verdadera naturaleza en el plano comunicativo.

De acuerdo con nuestras expectativas, hemos verificado que las FR de reproche se suelen emplear más comúnmente en intervenciones complejas — con tres o más enunciados-, pero también en intervenciones bimembres. Dicho esto, las intervenciones simples — con la FR como el único elemento - son, sin duda alguna, las menos frecuentes en el corpus consultado. Asimismo, hemos comprobado que la posición más frecuente de las FR dentro de dichas intervenciones complejas es la medial, por lo que las dos hipótesis de partida quedan corroboradas.

Del mismo modo, hemos visto que en la mayoría de los casos la FR va precedida por un enunciado (o un conjunto de enunciados) con distintos valores: un argumento, un juicio de valor, una expresión de enfado/irritación, una ofensa, etc. Lo mismo ocurre con los enunciados posteriores donde destacan las muestras del estado de ánimo del hablante — normalmente negativo — o, en general, su rechazo hacia las acciones previas del oyente. Independientemente del valor específico de los enunciados próximos a la FR, creemos que todos ellos tienen un mismo objetivo: reforzar la FR y su propio valor, tanto a nivel afectivo (expresivo), como a nivel exhortativo (directivo), cumpliendo así con la naturaleza dual del reproche como acto de habla. Así pues, podemos reorganizar el orden de los enunciados en las posiciones iniciales y finales dentro del esquema presentado en el apartado 1 (Ilustración 1), pero en esencia la estructura informativa queda intacta.

A pesar de los datos concluyentes a los que hemos llegado, creemos que el estudio permite nuevas vías de investigación, sobre todo en cuanto a la metodología y a la tipología de las FR, aún por analizar. En primer lugar, es necesario ampliar el banco de FR, sean estas de reproche o de otros valores enunciativos. Es más, cuanto mayor sea el espectro funcional de estas expresiones, más información obtendremos sobre su comportamiento dentro de los entornos comunicativas en los que se desarrollan. A este respecto, sería interesante hacer un estudio contrastivo entre las FR que vehiculan actos descorteses (i.e. reproche) y actos corteses (i.e. agradecer), pero también valorar las diferencias y similitudes entre los actos que a priori comparten la misma proposición (i.e. reproche, crítica, reprimenda, etc.).

Por último, la incorporación de los ejemplos provenientes de los corpus orales nos serviría para confirmar las perspectivas aquí planteadas y también, siempre y cuando sea posible, ofrecer los datos de tipo sociolingüístico. Gracias a ello, podríamos completar algunas lagunas que todavía se mantienen en el estudio general de las fórmulas rutinarias.

\section{Referencias}

1. Alvarado Ortega, M. B. (2006). ¿Son las fórmulas rutinarias enunciados independientes? En Face, T. L. y Klee, C. A. (Eds.), Selected Proceedings of the 8th Hispanic Linguistics Symposium (pp. 214-220). Somerville, MA: Cascadilla Proceedings Project.

2. Alvarado Ortega, M. B. (2008). Las fórmulas rutinarias en el español actual. Tesis Doctoral. Alicante: Universidad de Alicante. http://rua.ua.es/dspace/handle/10045/7726.

3. Amigot Castillo, L. (2014). Las fórmulas rutinarias expresivas del alemán y del 
español: Estudio teórico y análisis pragmático comparado. Tesis Doctoral. Madrid: Universidad Complutense de Madrid, https://eprints.ucm.es/id/eprint/28135/.

4. Ariza, M. (1978). Contribución al estudio del orden de palabras en español. Anuario de Estudios Filológicos, 1, pp. 11-42.

5. Briz, A. (1998a). El español coloquial en la conversación. Esbozo de pragmagramática. Barcelona: Ariel.

6. Briz, A. (1998b). Los conectores pragmáticos en la conversación coloquial (II): El papel metadiscursivo. Español actual, 59, pp. 39-56.

7. Briz, A. (2000a). Las unidades de la conversación. RILCE, 16 (2), pp. 225-246.

8. Briz, A. (2000b). Turno y alternancia de turno en la conversación. Revista Argentina de Lingüística, 3, pp. 3-27.

9. Briz, A. (2003). Las unidades de la conversación: El acto. Girón Alconchel, J. L, Iglesias Recuero, S., Herrero Ruiz de Loizaga, F. J. y Narbona, A. (Eds.), Estudios ofrecidos al profesor José Jesús de Bustos Tovar (Vol. 2, pp. 953-968). Madrid: Universidad Complutense de Madrid, Servicio de Publicaciones.

10. Briz, A., y Grupo Val.Es.Co. (2000). ¿Cómo se comenta un texto coloquial? Barcelona: Ariel.

11. Briz, A., y Hidalgo, A. (1998). Conectores y estructura de la conversación. En Martín Zorraquino, Ma A. y Montolío, E. (Eds.), Marcadores del discurso. Teoría y análisis. (pp. 121-142). Madrid: Arco Libros.

12. Brown, P. y Levinson, S. (1978). Universals in language usage: Politeness phenomena. En Goody, E. (Ed.), Questions and politeness. Strategies in Social interaction (pp. 56-289). Cambridge: Cambridge University Press.

13. Brown, P. y Levinson, S. (1987). Politeness. Some universals in language usage. Cambridge: Cambridge University Press. https://doi.org/10.1017/CBO9780511813085

14. Casares, J. (1950). Introducción a la lexicografía. Madrid: Consejo Superior de Investigaciones Científicas.

15. Contreras, H. (1978). El orden de las palabras en español. Madrid: Cátedra.

16. Corpas Pastor, G. (1996). Manual de la fraseología española. Madrid: Gredos.

17. Cortés, L., y Camacho, M. (2005). Unidades de segmentación y marcadores del discurso. Madrid: Arco Libros.

18. EsTenTen18 (Spanish Web 2018),. (s. f.). https://app.sketchengine.eu/\#dashboard?corpname=preloaded\%2Festenten18_fl5

19. Fuentes, C. (2007). Sintaxis del enunciado: Los complementos periféricos. Madrid: Arco Libros.

20. Fuentes, C. (2014). Los límites del enunciado. Estudios de Lingüística del Español, 35, pp. 143-167.

21. Grupo Val.Es.Co. (2002). La transcripción de la lengua hablada. El sistema del grupo Val.Es.Co. Español Actual, 77, pp. 1-30.

22. Grupo Val.Es.Co. (2014). Las unidades del discurso oral. La propuesta Val.Es.Co de segmentación de la conversación (coloquial). Estudios de Lingüística del Español, 35 (1), pp. 11-71.

23. Halliday, M. (1967). Notes on transitivity and theme in English. Journal of Linguistics, 3 (4), pp. 119-244. https://doi.org/10.1017/S0022226700016613

24. Hernando, L. A. (2005). El orden de palabras en español. Revista de Filología, 23, 161-178.

25. Hidalgo, A., y Padilla, X. (2006). Bases para el análisis de las unidades menores del discurso oral: Los subactos. Oralia, 9, pp. 109-143.

26. Koch, P. y Oesterreicher, W. (1985). Sprache der Nähe-Sprache der Distanz Mündlichkeit und Schriftlichkeit im Spannungsfeld von Sprachtheorie und Sprachgeschichte. Romanistisches Jahrbuch, 36, pp. 15-43. https://doi.org/10.1515/9783110244922.15

27. Koch, P. y Oesterreicher, W. (2007 [1990]). Lengua hablada en la Romania: Español, francés, italiano. Madrid: Gredos.

28. Koch, P. y Oesterreicher, W. (2001). Langage parlé et langage écrit. Lexikon der 
29. Latorre Garcia, L. (2018). Las unidades mínimas de discurso y las estructuras más frecuentes en la conversación coloquial. Normas, 8, pp. 10-24. https://doi.org/10.7203/Normas.v8i1.13323

30. López Serena, A. (2012). En los márgenes de la estructura informativa: La posición inicial de enunciado como «base». Lingüistica española actual, 34(2), pp. 303-337.

31. Núñez Bayo, Z. (2016). Las fórmulas oraciones en el español coloquial. Tesis doctoral. Alcalá de Henares: Universidad de Alcalá.

32. Padilla, X. (2005). Pragmática del orden de palabras. Alicante: Universidad de Alicante.

33. Padilla, X. (2006). Las unidades monológicas (intervención, acto y subacto) y el orden de palabras: Una guía para su análisis en el discurso hablado. En Casado Velarde, M., González Ruiz, R. y Romero Gualda, M. V. (Eds.), Análisis del discurso: Lengua, cultura, valores. (Vol. 1, pp. 677-690). Madrid: Arco Libros.

34. Portolés, J. (2010). Los marcadores del discurso y la estructura informativa. En Loureda, Ó. y Acín, E. (Eds.), Los estudios sobre marcadores del discurso, hoy (pp. 281-325). Madrid: Arco Libros.

35. Robles Sabater, F. y Bertomeu Pi, P. (2018). La estructura informativa en español hablado: Aproximación preliminar. Normas, 8(1), 1. https://doi.org/10.7203/Normas.v8i1.13500

36. Salameh, S., Estellés, M., y Pons, S. (2018). Beyond the notion of periphery: An account of polyfunctional discourse markers within the Val.Es.Co. Model of discourse segmentation. En Beeching, K. et al. (Eds.), Positioning the self and others (pp. 105-125). Amsterdam: John Benjamins. https://doi.org/10.1075/pbns.292.05sal

37. Searle, J. R. (1969). Actos de habla. Madrid: Cátedra.

38. Searle, J. R. (1976). A classification of illocutionary acts. Language in Society, 5(1), pp. 1-23. https://doi.org/10.1017/S0047404500006837

39. Searle, J. R. (1979). Expression and meaning. Studies in the Theory of Speech Acts. Cambridge: Cambridge University Press. https://doi.org/10.1017/CBO9780511609213

40. Seco, M., Andrés, O., y Ramos, G. (2009). Diccionario Fraseológico Documentado del Español Actual. Locuciones y modismos españoles. Madrid: Aguilar Lexicografía.

41. Tulimirovic, B. (2021a): Las fórmulas fraseológicas de reproche en español. Tesis doctoral, Universidad de Granada. En línea. https://digibug.ugr.es/handle/10481/67819?locale-attribute=fr

42. Tulimirovic, B. (2021b): Las fórmulas rutinarias al servicio del acto de habla del reproche: el caso de zapatero, a tus zapatos. Revista de investigación lingüística, 24, 235-253.

43. Vallejo Zapata, V. J. (2021). Caracterización de la fuerza ilocucionaria múltiple en el marco de la teoría de los actos de habla. Tesis doctoral. Antioquia: Universidad de Antioquia. <http://bibliotecadigital.udea.edu.co/handle/10495/19283>

44. Varela, F., y Kubarth, H. (2004). Diccionario Fraseológico del Español Moderno. Madrid: Gredos.

45. Vela Delfa, C., y Jiménez Gómez, J. J. (2011). El sistema de alternancia de turnos en los intercambios sincrónicos mediatizados por ordenador. Pragmalingüística, 19, pp. 121-138. https://doi.org/10.25267/Pragmalinguistica.2011.i19.08

46. Villalba, X. (2010). El orden de las palabras en español. Barcelona: Castalia. 